VoL. 50 (1994) [13-19]

\title{
ON THE MEROMORPHIC SOLUTIONS OF SOME FUNCTIONAL EQUATIONS
}

\author{
Jianhua Chen, Jianyong Qiao and Wenjun Zhang
}

Let $f(z)$ be a meromorphic function, let $P(z)$ and $Q(z)$ be two polynomials. We shall investigate the asymptotic behaviour of the ratio $T(r, f(P)) / T(r, f(Q))$, and discuss the growth of the meromorphic solutions of some functional equations.

\section{INTRODUCTION AND MAIN RESULTS}

Let $f(z)$ be a meromorphic function in $\mathbf{C}$. We denote the order and the lower order of $f(z)$ by $p_{f}$ and $\mu_{f}$ respectively.

There are many interesting works about the meromorphic solutions of functional equations(see $[4,5,7,8]$ et cetera). In this paper, we deal with the following functional equation:

where

$$
R_{1}(z, f(g(z)))=R_{2}\left(z, f\left(P_{m}(z)\right)\right)
$$

and

$$
R_{j}(z, w)=P_{j}(z, w) / Q_{j}(z, w),
$$

$$
P_{j}(z, w)=\sum_{i=0}^{p_{j}} a_{i j}(z) w^{i}, \quad Q_{j}(z, w)=\sum_{k=0}^{q_{j}} b_{k j}(z) w^{k}
$$

are two polynomials of $w$ which are mutually prime, $a_{i j}(z)$ and $b_{k j}(z)$ all are polynomials of $z ; f(w)$ is a transcendental meromorphic function; $g(z)$ is an entire function; $P_{m}(z)=a_{m} z^{m}+\cdots+a_{1} z+a_{0}\left(a_{m} \neq 0\right)$ is a polynomial of degree $m$. Put $\partial R_{j}=\max \left(p_{j}, q_{j}\right)(j=1,2)$. We have the following:

THEOREM 1. Let $R_{j}(z, w)(j=1,2), f(w), g(z)$ and $P_{m}(z)$ satisfy the equation (1). Then $g(z)$ is a polynomial and its degree $n$ lies between $m$ and $\left(\partial R_{2} / \partial R_{1}\right) m$. Furthermore, put $g(z)=b_{n} z^{n}+\cdots+b_{1} z+b_{0}\left(b_{n} \neq 0\right)$, We have

(1) If $m \neq n$, then $\rho_{f}=0$;

(2) If $m=n$ and $\left|a_{m}\right| \neq\left|b_{n}\right|$, then $\rho_{f}=\mu_{f}=\log \left(\partial R_{1}\right) /\left(\partial R_{2}\right) / \log \left|a_{m} / b_{n}\right|$;

(3) If $m=n,\left|a_{m}\right|=\left|b_{n}\right|$, and $\partial R_{1} \neq \partial R_{2}$, then $\mu_{f}=\infty$.

Received 18th August, 1993

Copyright Clearance Centre, Inc. Serial-fee code: 0004-9729/94 \$A2.00+0.00. 
Remarks. (1) Let $f$ be a meromorphic function, $g$ be an entire function, $P$ be a polynomial of degree $k$, and $f(g(z))=f(P(z))$. Gross [2, p.542, Problem 31] has posed the following problem: Must $g$ also be a polynomial of degree $k$ ? The above Theorem 1 can give an affirmative answer to this problem.

(2) In [5], Shimomura investigated the Schröder equation

$$
f(c z)=Q(f(z)),
$$

where $c$ is a constant with $|c|>1, Q(w)$ is a polynomial of degree $n$. He proved that any non-constant entire solution $f(z)$ of $(2)$ has order $\rho_{f}=\log n / \log |c|$. The above Theorem 1 is a generalisation of this result.

(3) In [8], Yanagihara investigated the following functional equation:

$$
f(z+1)=R(z, f(z))
$$

where $R(z, w)$ is a rational function of two variables. He proved that any transcendental meromorphic solution $f(z)$ of (3) has order $\rho_{f}=\infty$ if $\partial R>1$. The above Theorem 1 is a generalisation of this result.

Qiao [4] has investigated the asymptotic behaviour of the ratio

$$
T(r, f(\alpha z+\beta)) / T(r, f(z)) \text {. }
$$

Let $P_{m}(z)=a_{m} z^{m}+\cdots+a_{1} z+a_{0}$ and $Q_{n}(z)=b_{n} z^{n}+\cdots+b_{1} z+b_{0}\left(a_{m} b_{n} \neq 0\right)$ be two polynomials. Denote

$$
\rho_{f}^{*}=\varlimsup_{r \rightarrow \infty} \log T(r, f) / \log \log r ; \mu_{f}^{*}=\varliminf_{r \rightarrow \infty} \log T(r, f) / \log \log r .
$$

In this paper, we deal with the ratio

$$
\sigma\left(r, f, P_{m}, Q_{n}\right)=T\left(r, f\left(P_{m}\right)\right) / T\left(r, f\left(Q_{n}\right)\right),
$$

and prove the following:

THEOREM 2. (1) If $m>n$, then

$$
\varliminf_{r \rightarrow \infty} \sigma\left(r, f, P_{m}, Q_{n}\right) \leqslant\left(\frac{m}{n}\right)^{\mu_{f}^{*}} \leqslant\left(\frac{m}{n}\right)^{\rho_{f}^{*}} \leqslant \varlimsup_{r \rightarrow \infty} \sigma\left(r, f, P_{m}, Q_{n}\right) ;
$$

(2) If $m=n$, and $\left|a_{m}\right|>\left|b_{n}\right|$, then

$$
\varliminf_{r \rightarrow \infty} \sigma\left(r, f, P_{m}, Q_{n}\right) \leqslant\left|\frac{a_{m}}{b_{n}}\right|^{\mu_{f}} \leqslant\left|\frac{a_{m}}{b_{n}}\right|^{\rho_{f}} \leqslant \varlimsup_{r \rightarrow \infty} \sigma\left(r, f, P_{m}, Q_{n}\right) ;
$$

(3) If $m=n,\left|a_{m}\right|=\left|b_{n}\right|$, and $\mu_{f}<\infty$, then

$$
\varliminf_{r \rightarrow \infty} \sigma\left(r, f, P_{m}, Q_{n}\right) \leqslant 1 \leqslant \varlimsup_{r \rightarrow \infty} \sigma\left(r, f, P_{m}, Q_{n}\right) .
$$

REMARK. The proof of Theorem 1 mainly depends on Theorem 2. 


\section{The Proof of Theorem 2}

Put

$$
\Omega=\varliminf_{r \rightarrow \infty} \sigma\left(r, f, P_{m}, Q_{n}\right)
$$

Firstly, we prove that $(m / n)^{\mu_{f}^{*}},\left|a_{m} / b_{n}\right|^{\mu_{f}}$ and 1 are the upper bounds of $\Omega$ in the cases (1), (2) and (3) respectively. If $\Omega=0$, this is obviously true. Below, we suppose $\Omega>0$ ( $\Omega$ may be infinity). Therefore, for any finite and positive number $\tau<\Omega$, there exists $r_{1}>0$, such that

$$
T\left(r, f\left(P_{m}\right)\right)>\tau \cdot T\left(r, f\left(Q_{n}\right)\right)
$$

when $r \geqslant r_{1}$. Choose a complex number $a$ which isnt a Valiron deficient value of $f\left(P_{m}\right), f\left(Q_{n}\right)$ and $f(z)$. Thus for any $\varepsilon>0$, from the above inequality we deduce that there exists some $r_{2}>r_{1}$, such that

$$
N\left(r, f\left(P_{m}\right)=a\right)>\tau \cdot \frac{1-\varepsilon}{1+\varepsilon} N\left(r, f\left(Q_{n}\right)=a\right)
$$

when $r \geqslant r_{2}$.

Now $\left|Q_{n}(z)\right| \sim\left|b_{n}\right||z|^{n}$ as $z \rightarrow \infty$. For a positive number $\delta<\min \left(\left|a_{m}\right|,\left|b_{n}\right|\right)$ , put $A_{1}=\left|b_{n}\right|-\delta$, and then there exists $R>0$ such that $\left|Q_{n}(z)\right| \geqslant A_{1}|z|^{n}$ when $|z| \geqslant R$. Therefore, all roots of $Q_{n}(z)=w$ must lie in $\{z:|z|<r\}$ when $r \geqslant R$ and $|w|<A_{1} r^{n}$. This means that $n\left(r, Q_{n}=w\right)=n$ when $r \geqslant R$ and $|w|<A_{1} r^{n}$. Denote the roots of $f(w)=a$ by $\left\{w_{k}\right\}$. Thus

$$
\begin{aligned}
n\left(r, f\left(Q_{n}\right)\right) & =\sum_{\left|w_{k}\right| \leqslant M\left(r, Q_{n}\right)} n\left(r, Q_{n}=w_{k}\right) \geqslant \sum_{\left|w_{k}\right|<A_{1} r^{n}} n\left(r, Q_{n}=w_{k}\right) \\
& =n \cdot n\left(A_{1} r^{n}, f=a\right)
\end{aligned}
$$

when $r \geqslant R$. It follows that

$$
\begin{aligned}
N\left(r, f\left(Q_{n}\right)=a\right) & =\int_{0}^{r} \frac{n\left(t, f\left(Q_{n}\right)=a\right)-n\left(0, f\left(Q_{n}\right)=a\right)}{t} d t \\
& \geqslant \int_{R}^{r} \frac{n\left(t, f\left(Q_{n}\right)=a\right)}{t} d t+O(1) \\
& \geqslant \int_{R}^{r} \frac{n \cdot n\left(A_{1} t^{n}, f=a\right)}{t} d t+O(1)=\int_{A_{1} R^{n}}^{A_{1} r^{n}} \frac{n(t, f=a)}{t} d t+O(1) \\
& =N\left(A_{1} r^{n}, f=a\right)-N\left(A_{1} R^{n}, f=a\right)+O(1)
\end{aligned}
$$

We thus obtain

$$
N\left(r, f\left(Q_{n}\right)=a\right) \geqslant N\left(A_{1} r^{n}, f=a\right)+O(1),(r \rightarrow \infty)
$$


On the other hand, for sufficiently large $r$, we have

$$
n\left(r, f\left(P_{m}\right)=a\right)=\sum_{\left|w_{k}\right| \leqslant M\left(r, P_{m}\right)} n\left(r, P_{m}=w_{k}\right) \leqslant m \cdot n\left(A_{2} r^{m}, f=a\right),
$$

where $A_{2}=\left|a_{m}\right|+\delta$. It follows that

$$
N\left(r, f\left(P_{m}\right)=a\right) \leqslant N\left(A_{2} r^{m}, f=a\right)+O(1),(r \rightarrow \infty) .
$$

Since $a$ isnt a Valiron deficient value of $f(z)$, it follows from (7), (8) and (9) that there exists $r_{3}>r_{2}$, such that

$$
T\left(A_{2} r^{m}, f\right) \geqslant\left(\frac{1-\varepsilon}{1+\varepsilon}\right)^{3} \tau T\left(A_{1} r^{n}, f\right)
$$

when $r \geqslant r_{3}$. Put $c=((1-\varepsilon) /(1+\varepsilon))^{3} \tau, t=A_{2} / A_{1}^{m / n}$, and $R_{1}=A_{1} r_{3}^{n}$. Hence it follows from (10) that

$$
T\left(t \dot{r}^{m / n}, f\right) \geqslant c T(r, f),\left(r \geqslant R_{1}\right)
$$

We discuss the following three cases:

(1) If $m>n$. For any $\varepsilon>0$, put $\alpha=m / n+\varepsilon$ and assume $t / r^{e}<1$ when $r \geqslant R_{1}$ (otherwise, we choose a larger $R_{1}$ ). By (11), we obtain that

$$
T\left(r^{\alpha}, f\right) \geqslant c_{1} T(r, f),\left(r \geqslant R_{1}\right) .
$$

It follows that

$$
T\left(R_{1}^{\alpha^{k}}, f\right) \geqslant c_{1}^{k} T\left(R_{1}, f\right),(k=1,2,3, \cdots)
$$

For arbitrary real number $r \geqslant R_{1}$, since $\alpha>1$, we assume $r \in\left[R_{1}^{\alpha^{p}}, R_{1}^{\alpha^{p+1}}\right)$ for some natural number $p$. By (12) we deduce

$$
T(r, f) \geqslant T\left(R_{1}^{\alpha^{p}}, f\right) \geqslant c^{p} T\left(R_{1}, f\right)>\lambda_{1} c^{\log \log r / \log \alpha}
$$

where $\lambda_{1}$ is a positive number. It follows immediately that $\mu_{f}^{*} \geqslant \log c / \log \alpha$. Let $\varepsilon \rightarrow 0$, then $c \rightarrow \tau, \alpha \rightarrow m / n$. Thus $\tau \leqslant(m / n)^{\mu_{j}^{*}}$. Let $\tau \rightarrow \Omega$, then $\Omega \leqslant(m / n)^{\mu_{j}^{*}}$.

(2) If $m=n$ and $\left|a_{m}\right|>\left|b_{n}\right|$. Let $\delta$ be sufficiently small such that $A_{2}>A_{1}$. Since $t>1$, it follows from (11) that

$$
T\left(t^{k} R_{1}, f\right) \geqslant c^{k} T\left(R_{1}, f\right),(k=1,2,3, \cdots) .
$$


For arbitrary real number $r \geqslant R_{2}$, we assume $r \in\left[R_{1} t^{p}, R_{1} t^{p+1}\right)$ for some natural number $p$. By (13) we deduce

$$
T(r, f) \geqslant T\left(R_{1} t^{p}, f\right) \geqslant c^{p} T\left(R_{1}, f\right)>\mu_{1} c^{\log r / \log t},
$$

where $\mu_{1}$ is a positive number. It follows that $\mu_{f} \geqslant \log c / \log t$. Let $\varepsilon \rightarrow 0$, then $c \rightarrow \tau$, and $t \rightarrow\left|a_{m} / b_{n}\right|$. Thus $\tau \leqslant\left|a_{m} / b_{n}\right|^{\mu f}$. Let $\tau \rightarrow \Omega$, then $\Omega \leqslant\left|a_{m} / b_{n}\right|^{\mu f}$.

(3) If $m=n,\left|a_{m}\right|=\left|b_{n}\right|$ and $\mu_{f}<\infty$. Since $A_{2}>A_{1}$, we have $t>1$. We can deduce $\mu_{f} \geqslant \log c / \log t$ by the same method as in case 2). Let $\varepsilon \rightarrow 0$, then $c \rightarrow \tau$ and $t \rightarrow 1$. Thus $\tau \leqslant 1$. Let $\tau \rightarrow \Omega$, then $\Omega \leqslant 1$.

Now

$$
\varlimsup_{r \rightarrow \infty} \sigma\left(r, f, P_{m}, Q_{n}\right)=1 /\left(\varliminf_{r \rightarrow \infty} \sigma\left(r, f, Q_{n}, P_{m}\right)\right)
$$

Therefore, by the above discussion, we know that $\varlimsup_{r \rightarrow \infty} \sigma\left(r, f, P_{m}, Q_{n}\right)$ has the lower bounds as stated in Theorem 2. The proof of Theorem 2 is thus complete.

\section{The proof of Theorem 1}

In order to prove Theorem 1, we need the following results:

LEMMA 1. [3] Let $g(z)$ be a transcendental entire function, $q$ be a natural number. Then for any $M>0$, there exists $R_{0}>0$ and $R_{n} \rightarrow \infty$ (here $R_{0}<R_{1}<R_{2}<$ $\left.\cdots<R_{n}<\cdots\right)$, such that

$$
N(r, g(z)=w)>M
$$

when $r \in\left[R_{n}, R_{n}^{2}\right]$ and $w \in\left\{w: R_{0} \leqslant|w| \leqslant r^{q}\right\}$.

LEMma 2. Let $g(z)$ be a transcendental entire function, $P(z)$ be a polynomial and $f(w)$ be a meromorphic function. Then

$$
\varlimsup_{r \rightarrow \infty} T(r, f(g)) / T(r, f(P))=\infty
$$

Proof: Denote the degree of $P(z)$ by $m$. We choose a natural number $q>m$ and real number $M>0$. By Lemma 1 , there exist $R_{0}$ and $R_{n} \rightarrow \infty$ (here $R_{0}<$ $\left.R_{1}<R_{2}<\cdots<R_{n}<\cdots\right)$, such that the inequality (14) holds when $r \in\left[R_{n}, R_{n}^{2}\right]$ and $w \in\left\{w: R_{0} \leqslant|w| \leqslant r^{q}\right\}$. Now we can deduce the following inequality by a method similar to that used in [1] to prove that the superior limit of $T(r, f(g)) / T^{\prime}(r, f)$ is infinite:

$$
T\left(R_{n}^{2}, f(g)\right)>\frac{M}{2 q}(1+o(1)) T\left(R_{n}^{2 q}, f(z)\right),(n \rightarrow \infty)
$$


Choose a complex number $a$ which is not a Valiron deficient value of $f(g)$. We deduce from (10) and (15) that

$$
T\left(R_{n}^{2}, f(g)\right)>\frac{M}{2 q}(1+o(1)) T\left(R_{n}^{2}, f(P)\right),(n \rightarrow \infty) .
$$

Let $M \rightarrow \infty$, then the proof of Lemma 2 is complete.

LEMMA 3. [6] Let $R(z, w)$ be a rational function of two variables, and let $f$ be a meromorphic function. Then

$$
T(r, R(z, f(z)))=\partial R \cdot T(r, f)+O(\log r),(r \rightarrow \infty)
$$

The Proof of Theorem 1: Firstly, we can deduce from the equality (1) and Lemma 3 that

$$
\lim _{r \rightarrow \infty} T(r, f(g)) / T\left(r, f\left(P_{m}\right)\right)=\frac{\partial R_{2}}{\partial R_{1}} \neq \infty .
$$

It follows from Lemma 2 that $g(z)$ is a polynomial. If the degree $n$ of $g(z)$ is not equal to $m$, by Theorem 2 and (16) we obtain

$$
(n / m)^{\rho_{f}^{*}}=\frac{\partial R_{2}}{\partial R_{1}}
$$

Since $f$ is not a constant, we have $\rho_{f}^{*} \geqslant 1$. By (17) we know : If $n<m$, then $n / m \geqslant(n / m)^{\rho_{f}^{*}}=\left(\partial R_{2}\right) /\left(\partial R_{1}\right)$, thus $n \geqslant\left(\left(\partial R_{2}\right) /\left(\partial R_{1}\right)\right) \cdot m ;$ If $n>m$, then $n / m \leqslant$ $(n / m)^{\rho_{f}^{*}}=\left(\partial R_{2}\right) /\left(\partial R_{1}\right)$, thus $n \leqslant\left(\left(\partial R_{2}\right) /\left(\partial R_{1}\right)\right) \cdot m$. Hence $n$ lies between $m$ and $\left(\left(\partial R_{2}\right) /\left(\partial R_{1}\right)\right) \cdot m$. Below, we discuss three cases:

(1) If $m \neq n,(17)$ implies $\rho_{f}^{*}<\infty$, thus $\rho_{f}=0$.

(2) If $m=n$ and $\left|a_{m}\right| \neq\left|b_{n}\right|$, without loss of generality, we suppose $\left|a_{m}\right|>$ $\left|b_{n}\right|$. By Theorem 2 and (16),

$$
\left|\frac{a_{m}}{b_{n}}\right|^{\mu_{f}}=\left|\frac{a_{m}}{b_{n}}\right|^{\rho_{f}}=\frac{\partial R_{1}}{\partial R_{2}} .
$$

Hence $\partial R_{1} \geqslant \partial R_{2}$ and

$$
\rho_{f}=\mu_{f}=\log \frac{\partial R_{1}}{\partial R_{2}} / \log \left|\frac{a_{m}}{b_{n}}\right| .
$$

(3) If $m=n,\left|a_{m}\right|=\left|b_{n}\right|$ and $\partial R_{1} \neq \partial R_{2}$, by Theorem 2 and (16) we have $\mu_{f}=\infty$. The proof of Theorem 1 is complete. 


\section{REFERENCES}

[1] J. Clunie, 'The composition of entire and meromorphic function', in Mathematical Essays Dedicated to A.J. Mclntyre (Ohio University Press, Athens, Ohio, 1970), pp. 75-92.

[2] J. Korevaar, Entire functions and related parts of analysig, Proceedings of symposia in pure mathematics XI (Amer. Math. Soc., 1968).

[3] K. Niino and N. Suita, 'Growth of a composite function of entire functions', Kodai Math. J. 3 (1980), 374-379.

[4] J. Qiao, 'On the growth of compositions of linear and meromorphic functions', Bull. Austral. Math. Soc. 44 (1991), 263-269.

[5] S. Shimomura, 'Entire solutions of a polynomial difference equation', J. Fac. Sci. Univ. Tokyo Sect. 1 A Math. 28 (1981), 253-266.

[6] G. Valiron, 'Sur la dérivée des fonctoions algébroïdes', Bull. Soc. Math. France 59 (1931), 17-39.

[7] N. Yanagihara, 'Meromorphic solutions of some difference equations', Funkcial Ekva. 23 (1980), 309-326.

[8] N. Yanagihara, 'Meromorphic solutions of some functional equations', Bull. Sci. Math. 107 (1983), 289-300.

Institute of Mathematics

Huaibei Coal Industry Teachers College

Huaibei, Anhui Province

People's Republic of China

Institute of Mathematics

Fudan University

Shanghai

People's Republic of China
Institute of Mathematics

Huaibei Coal Industry Teachers College

Huaibei, Anhui Province

People's Republic of China 